\title{
CAPACIDADES PARA TRABALHAR COM ALIANÇA: UM ESTUDO DE CASO LONGITUDINAL
}

\section{ABILITY TO WORK WITH ALLIANCES: A LONGITUDINAL CASE STUDY}

\author{
Gustavo Palmisano Avelar \\ Fundação Dom Cabral - FDC \\ gustavo@fdc.org.br \\ Rosileia Milagres \\ Fundação Dom Cabral - FDC \\ rosileiam@fdc.org.br
}

Submissão: $21 / 11 / 2019$

Aprovação: 19/08/2020

\begin{abstract}
RESUMO
Este artigo identificou vantagens e desvantagens da implementação de uma estrutura dedicada à gestão de alianças, assim como avaliou os instrumentos criados e sua finalidade. Os resultados demonstram que as vantagens da estrutura dedicada - mediação, organização e centralização da relação - superam as desvantagens - burocratização e lentidão no processo de tomada de decisões. A pesquisa contribuiu para a discussão sobre o papel do escritório e as relações entre ele e os mecanismos de formação das capacidades de aliança. Reafirmou sua importância no que se refere ao acúmulo, troca de conhecimento e identificação de oportunidades, confirmando a literatura. Outra contribuição relaciona-se à avaliação longitudinal - método pouco utilizado na literatura até então - da atuação do escritório elucidando sua evolução e mecanismos de aprendizado. No que se refere à prática gerencial, o estudo contribuiu para demonstrar a necessidade de autonomia, apoio organizacional ao escritório e de treinamento específico em gestão de portfólio de alianças para os gestores envolvidos na coordenação de alianças.
\end{abstract}

Palavras-chave: Alianças estratégicas; Capacidades de aliança; Estratégia colaborativa; Parcerias; Arranjos colaborativos.

\begin{abstract}
This article has identified the advantages and disadvantages of implementing a company structure dedicated to managing alliances and has also evaluated the instruments created and their purposes. The results demonstrate that the advantages of a dedicated structure mediation, organization and relationship centralization - outweigh the disadvantages bureaucratization and slow decision-making. The research has contributed to the discussion regarding the role of this office and the relationship between itself and the mechanisms for forming the alliances capabilities. The research has reaffirmed its importance in regards to accumulation, exchange of knowledge and identification of opportunities, according to what
\end{abstract}


the literature has presented. Another contribution is related to the longitudinal evaluation - a method little used in the literature until now - of the office's performance - elucidating its evolution and learning mechanisms. Regarding managerial practice, the study has contributed to demonstrate the need for autonomy, organizational support for the office and specific training in alliance portfolio management for those managers involved in coordinating alliances.

Keywords: Strategic alliances; Alliance capabilities; Collaborative strategy; Partnerships; Collaborative Arrangements.

\section{INTRODUÇÃO}

No início da década de 1990, houve um significativo aumento na formação de alianças estratégicas, com crescimento de aproximadamente $300 \%$ entre os anos de 1990 e 1995 (Schilling, 2015). Desde então, muito tem sido escrito sobre sua proliferação e como se tornaram importantes nas estratégias de crescimento e competitividade das empresas (Kale \& Singh, 2009; Moller, 2013). Entretanto, apesar desse aumento, vários estudos mostram que quase metade das alianças formadas não obtém sucesso (Kale, Dyer, \& Singh, 2002). Dentre aquelas bem-sucedidas estão as que conseguem formar capacidades de aliança (Anand \& Khanna, 2000; Kale et al., 2002; Kale \& Singh, 2009; Schreiner; Kale, \& Corststen, 2009; Moller, 2013; Niesten \& Jolink, 2015).

Essas capacidades podem ser definidas como as habilidades das empresas para capturar, compartilhar e armazenar o conhecimento adquirido em um processo específico e utilizá-lo em outras alianças (Kale et al., 2002; Heimeriks \& Duyster, 2007; Kale \& Singh, 2007). Para sua formação, Heimeriks et al. (2009) e Sluyts, Martens and Matthyssens (2008) defendem que os mecanismos de transferência de conhecimento são importante fator na mediação entre experiência e formação de capacidades de aliança, pois capturam e replicam os conhecimentos gerados, levando ao aprendizado e sistematização do conhecimento.

Com o objetivo de capturar, armazenar e aplicar esse conhecimento, as empresas criam departamentos/ áreas e alocam times dedicados à gestão do seu portfólio de alianças (Heimeriks \& Duysters, 2007; Kale et al., 2002). Essas estruturas ajudam a construir as capacidades de aliança porque facilitam a efetiva coordenação dos assuntos relacionados às parcerias e servem de depósito para o conhecimento gerado (Kale et al., 2002).

A literatura se alinha sobre a importância dessas estruturas (Kohtamaki, Rabetino, \& Moller, 2018), afirmando que permitem a gestão do portfólio de alianças, a troca de informações e a avaliação de novas oportunidades aderentes à estratégia das organizações (Kale et al., 2002; Zollo \& Winter, 2002; Draulans, DeMan, \& Volberda, 2003; Dyer, Kale, \& Singh, 2001; Heimeriks \& Duysters, 2007; Kale \& Singh, 2007; Heimeriks et al., 2009). Esta capacidade possui efeitos significativos na co-criação de valor, inovação e na performance ao longo da cadeia produtiva (Niesten \& Jolink, 2015). Segundo a revisão de literatura realizada por Kohtamaki, Rabetino e Moller (2018) os estudos sobre capacidades de aliança são relativamente recentes, sendo o primeiro artigo publicado no final dos anos 1990. A maior parte dos artigos foi publicada entre 2005 e 2017, sendo a minoria apoiada em base empírica e dentre estes, poucos utilizaram métodos qualitativos. Ressente-se de mais estudos qualitativos que permitam um melhor entendimento das micro-atividades que formam as capacidades de aliança. Perguntas como o que os gestores fazem quando gerenciam e desenvolvem relações de parceria; quais são as práticas e práxis utilizadas; como as rotinas, processos e outras atividades suportam o aprendizado ao longo do processo, dentre outras, são citadas como espaços abertos para maiores investigações. Há também uma lacuna na avaliação das 
desvantagens trazidas pela utilização destas estruturas, uma vez que a maior parte dos estudos se concentra em suas vantagens.

Neste sentido, este artigo visa debater as vantagens e desvantagens provenientes do estabelecimento de um escritório de alianças. Quais são os ganhos vislumbrados e quais são os efeitos indesejados? Quais os mecanismos de gestão criados por essa estrutura e com que finalidade? Procurou-se respondê-las por meio de um estudo de caso longitudinal. Conforme a revisão de literatura realizada por Kohtamaki et al., (2018) e o artigo de Tzokas, Kim, Akbar e Al-Dajani (2015) há necessidade de mais estudos qualitativos longitudinais que explorem os processos e atividades necessários para criar, gerenciar, integrar e aprender em ambientes de alianças.

Segundo os entrevistados, a estrutura dedicada à gestão dos parceiros contribuiu para o desenvolvimento da parceria, pois propiciou a centralização da demanda, mediação e organização do relacionamento entre as partes. A principal crítica, por sua vez, está relacionada à lentidão na resolução dos problemas.

$\mathrm{O}$ estudo mostra que essa estrutura implementou, ainda que de maneira parcial e/ou com alterações de escopo em relação às melhores práticas apontadas na literatura, quatro dos onze mecanismos descritos por Heimeriks et al. (2009): sessões conjuntas de planejamento; registro de melhores práticas; intranet para gestão de recursos das alianças e programas de treinamento in house.

Entretanto, a simples existência dessa estrutura não garantiu a implementação desses mecanismos de forma consistente ao longo do tempo. A análise longitudinal mostrou que os mecanismos de formação das capacidades de aliança inicialmente implementados foram gradativamente abandonados, com a percepção entre as partes de diminuição do espírito de cooperação.

\section{REFERENCIAL TEÓRICO}

Visando a manter suas posições competitivas, as organizações utilizam cada vez mais estratégias de cooperação (Kale \& Singh, 2009). Elas têm sido um ponto central para segmentos como telecomunicações e farmacêutico (Cools \& Roos, 2005, Figgis et al., 2017). Relatório de pesquisa da Price Waterhouse Coopers (PwC) em 2017 indica que elas continuam sendo uma dentre as três prioridades mais citadas por Chief Executive officer's (CEO's) de forma global (48\% de menções).

Sua expansão ganhou força a partir de meados dos anos 90, quando as alterações do ambiente de negócios, o surgimento de novos produtos, o encurtamento dos ciclos de inovação e as mudanças tecnológicas redesenharam os limites das organizações (Gulati, 1995; Little, 2001; Sluyts et al., 2008). São vistas como alternativa flexível para acesso a recursos complementares e competências fora dos limites da companhia, tornando-se importante escolha estratégica para alcançar vantagem competitiva (Dyer et al., 2001).

Milagres e Rezende (2014) indicam que, dentre as motivações para a adoção dessas estratégias, estariam a expansão da oferta de serviço/produto, acesso a mercados, obtenção de tecnologias e know-how, retenção de clientes, reduzção de custos, acesso a capacidades, obteção de economias de escala e redução do tempo de desenvolvimento de produtos/serviços. Por outro lado, muitos mostram que quase metade das alianças acabam não alcançando sucesso e que um percentual entre $48 \%$ e $53 \%$ de falhas acontece em um prazo de 24 meses. As razões para o insucesso são a falta de encaixe dos recursos complementares, incompatibilidade de culturas, processos de decisão diferentes, ausência de confiança, escolha 
inapropriada de estruturas de governança, inabilidade no gerenciamento de conflitos, mudanças no ambiente de negócios, entre outros (Kale et al., 2002).

Entretanto, Draulans et al. (2003) defendem que os fatores de sucesso das alianças não estão centrados em suas características intrínsecas, mas, na capacidade de gerenciamento. Pesquisas mostram que algumas empresas conseguem resultados superiores (Schreiner, Kale, \& Cortsten, 2009; Ireland, Hitt, \& Vaidyaniath; 2002), notadamente aquelas que possuem capacidades de aliança desenvolvidas (Anand \& Khanna, 2000; Kale et al. 2002, Heimeriks \& Duyster, 2007). Nesta linha de raciocínio, Duysters, Heimeriks, Lokshin, Meijer e Sabidussi (2012) sugerem o desenvolvimento das capacidades de aliança para gestão do seu portfólio.

Neste sentido, muitos estudos ressaltam a importância das funções dedicadas à gestão das alianças e o desenvolvimento de ferramentas e mecanismos específicos para o seu gerenciamento (Dyer et al., 2001; Heimeriks \& Duysters, 2007; Sluyts et al., 2010). Heimeriks et al. (2009) concordam que o desenvolvimento das capacidades de aliança demanda a existência de mecanismos e processos de gerenciamento que levem ao aprendizado e sistematização do conhecimento e, para tanto, agrupam estes mecanismos em quatro categorias:

1) Funções/estruturas específicas - estruturas especializadas para trabalharem diretamente na gestão e manutenção do portfólio de alianças. Têm, geralmente, uma estrutura de cargos, como vice-presidentes (VP) e gerentes de alianças.

2) Soluções baseadas em ferramentas - uma série de práticas, instrumentos, rotinas, regras, manuais, dentre outros, que provêm um guia que permite o aprendizado e aumentam o know how das organizações para lidarem com os desafios de gerir o portfólio de alianças da organização.

3) Programas de treinamento interno e externo - processos de compartilhamento de conhecimento que permitem insights sobre aspectos específicos do gerenciamento das alianças e estimulam as trocas de know how dentro das organizações.

4) Contratação de experts: utilização de terceiros como consultores, advogados, mediadores ou experts financeiros para complementar o conhecimento interno sobre o gerenciamento de parcerias.

Quadro 1.

Heimeriks et al. (2009) subdividiu essas quatro categorias em 14 itens, conforme

Quadro 1 - Atributos para a formação de capacidades de alianças

\begin{tabular}{|l|}
\hline \multicolumn{1}{|c|}{ CATEGORIA SOLUÇÃO } \\
\hline I. Estrutura Funcional e Staffing \\
\hline 1. Departamento de alianças \\
\hline 2. Vice Presidência de alianças \\
\hline 3. Gerente de alianças \\
\hline II. Ferramentas de Gestão de Alianças \\
\hline 4. Protocolo de seleção de parceiros \\
\hline 5. Sessões conjuntas de planejamento \\
\hline 6. Registro melhores práticas \\
\hline 7. Intranet para gestão recursos das alianças \\
\hline III. Treinamento \\
\hline 8. Programas in house \\
\hline
\end{tabular}




\begin{tabular}{|l|}
\hline 9. Programas de treinamento intercultural \\
\hline 10. Programas conduzidos por terceiros \\
\hline IV. Terceiros \\
\hline 11. Consultores \\
\hline 12. Experts legais \\
\hline 13. Mediadores \\
\hline 14. Experts Financeiros \\
\hline
\end{tabular}

Fonte: Heimeriks et al. (2009, p.8).

Funções/estruturas para gestão das alianças: A criação dessas estruturas permite a sistematização das experiências e é importante para capturar, integrar e disseminar o know how adquirido no gerenciamento das alianças (Kale et al., 2002) e criação de valor a partir do seu portfólio (Sarkar, Aulakh, \& Madhok, 2009). Podem ser formalizadas por meio da criação de departamentos e pessoal dedicado (Heimeriks \& Duysters, 2007; Kale et al., 2002). Facilitam a coordenação das questões relacionadas às alianças e são depositários do conhecimento gerado (Kale et al., 2002), levando à melhoria da performance das parcerias (Heimeriks et al., 2009). Ademais, aumentam a visibilidade externa, pois simbolizam o comprometimento com a estratégia de cooperação (Heimeriks et al., 2009).

Castro e Roldán (2015) afirmam que a coordenação do portfólio de alianças é o melhor preditor para uma melhor performance. Um estudo com 25 empresas europeias de grande porte mostra que a maioria delas havia criado posições específicas para a gestão de alianças, como sponsors, consultores internos e gestores das alianças (Sluyts et al., 2008). Milagres \& Resende (2014) reforçam a importância dessa estrutura ao identificarem em estudo de caso que ela é percebida como importante para disseminar os conhecimentos oriundos dos processos de parceria. Funciona, ainda, como referência para os gestores, diminuindo a incerteza em relação aos padrões de comportamentos que precisam ser adotados e, consequentemente, diminuindo os conflitos no âmbito das alianças e entre elas e as organizações.

Heimeriks, et al. (2015) afirmam que, apesar de fundamental, o timing da aliança influencia diretamente a adoção das estruturas de gestão. Para eles as empresas mais beneficiadas são aquelas que estão em fase de seleção de parceiros ou no momento de término das parcerias - na fase de implantação das alianças, elas são menos importantes.

Soluções baseadas em ferramentas: incluem manuais, checklists, guias, templates, rotinas, regras, bases de dados que sistematizam, compartilham, armazenam, permitem a aplicação do conhecimento codificado (Sluyts, Matthyssens, Martens, \& Streukens, 2011; Anand \& Khanna, 2000; Draulans et al., 2003; Heimeriks \& Duysters, 2007; Kale \& Singh, 2007) e apoiam as ações ou decisões a serem tomadas (Kale \& Singh, 2007)

A codificação tem foco no fornecimento de conteúdo (know-what), metodologia (know-how) e entendimento dos por quês (know-.why). Além disso, o conhecimento gerado cria uma memória para prevenir a perda de know-how por um eventual turnover de colaboradores (Sampson, 2007). Heimeriks et al. (2015) atestam que o ciclo de vida da aliança influencia a necessidade de mais ou menos ferramentas de codificação. Elas são mais benéficas nas fases de seleção de parceiros e encerramento dos acordos.

Programas de treinamento interno e externo. Os processos de treinamento internos ou externos contribuem para a troca, disseminação e internalização de conhecimento. (Kale \& Singh, 2007), sendo as comunidades de interação importantes veículos (Kale \& Singh, 2009). Sluyts et al. (2011) definem quatro etapas para o processo de aprendizado nas alianças: 
articulação, codificação, compartilhamento e internalização. Através da articulação de processos, como reports, apresentações e debriefings, os gestores são encorajados a tornar o seu conhecimento explícito. A codificação transforma esse conhecimento tácito em ferramentas escritas, templates e processos que suportam o dia a dia das alianças. $\mathrm{O}$ compartilhamento é a próxima etapa, realizado em encontros, sessões de brainstorm, fóruns, seminários etc.

E, por fim, os treinamentos formais que consolidam e internalizam os conhecimentos adquiridos. Os treinamentos tendem a ter mais sucesso para organizações com pouca experiência em processos de parceria. Draulans et al. (2003) e Heimeriks et al. (2009) defendem que as trocas entre pares em treinamentos formais ou rotinas de compartilhamento são reconhecidas como, possivelmente, a forma mais efetiva de intercâmbio. A literatura aponta que o desenvolvimento das habilidades, atitudes e conhecimentos dos gestores das alianças tende a melhorar os resultados (Sluyts et al., 2008).

Contratação de experts: A contratação de terceiros pode contribuir não somente para a solução do problema em si, mas, também, para mediar conflitos. Pode exercer o papel de agente independente, ser mais objetiva, trazer rigor ao processo de planejamento e assegurar que as metas definidas sejam realistas e promovidas de forma equânime entre os parceiros (Heimeriks et al., 2009). As organizações mais experientes com parceria tendem a se beneficiar mais com o uso de um especialista em alianças, seja ele terceiro ou colaborador (Draulans et al., 2003).

\section{METODOLOGIA}

O método de investigação baseou-se em estudo de caso único, de natureza qualitativa e perspectiva longitudinal (Yin, 2015). A escolha do caso atende às necessidades da pergunta estabelecida, como a existência de um escritório dedicado à gestão das parcerias e amplo acesso a documentos, gestores e parceiros que cubram o período analisado. Além disto, tratase de um método que permite o exame profundo, trazendo à tona especificidades do contexto investigado (Greenwood, 1973). Embora muito defendido pela literatura, o escritório dedicado não é uma realidade para algumas empresas brasileiras, mesmo nas de grande porte e/ou multinacionais que possuem um amplo conjunto de alianças. No processo de escolha do campo, foram avaliadas grandes organizações de setores diversos, como farmacêutico, indústria automotiva e tecnologia com um amplo portfólio de alianças, mas não foi encontrado um escritório dedicado.

A coleta partiu da análise de informações disponíveis em relatórios anuais, atas de reuniões, apresentações, livros escritos por executivos da empresa nódulo da parceria e material/documentos de treinamento. No total, foram analisadas 2.150 páginas. Estas múltiplas fontes de evidências permitem avaliar o mesmo fenômeno por vários ângulos e ajuda a reforçar a validade do constructo emergente do estudo de caso (Yin, 1981; Eisenhardt, 1989; Yin 2015). Em relação às entrevistas, foram trabalhados dois roteiros. O primeiro voltado para a empresa nódulo e o segundo para os parceiros. Nos dois, o papel do escritório era abordado, investigando suas vantagens e desvantagens, a criação dos mecanismos de gestão implementados e o desenvolvimento da atuação da estrutura ao longo do tempo. Foram realizadas 26 entrevistas virtuais por meio de ferramenta de comunicação eletrônica entre março e maio de 2018. As entrevistas foram gravadas e transcritas, gerando um documento de 190 páginas. As informações coletadas foram tratadas preservando a sua relevância e confidencialidade para as organizações envolvidas (FLICK, 2004).

Para analisar o caso, considerou-se as seguintes categorias analíticas: estrutura 
funcional, ferramentas de gestão de alianças, treinamento e contratação de terceiros (Heimeriks et all., 2009). Os autores dividiram as três últimas categorias em onze subcategorias, conforme apresentado no referencial teórico. O estudo de campo revelou a utilização de apenas quatro instrumentos, quais sejam: 1) sessões conjuntas de planejamento; 2) registro de melhores práticas, 3) intranet para gestão de recursos das alianças e 4) programas de treinamento in house.

Conforme proposto por Yin (2015), a análise das informações foi feita por meio da convergência de dados. Primeiramente, foi criada uma matriz de análise que posicionava em um eixo os respondentes divididos em dois grupos: gestores de empresa nódulo e parceiros. No outro estavam posicionadas as variáveis de análise: vantagens e desvantagens, a atuação do escritório nos marcos definidos e os mecanismos de gestão das alianças, conforme descritos por Heimeriks et al. (2009).

Para essa análise foi utilizada a ferramenta de planilhas eletrônicas Microsoft Excel. $\mathrm{Na}$ primeira etapa foram analisadas as citações por respondente horizontalmente, para identificar as menções individuais. As citações foram transcritas para a planilha de análise e, posteriormente, categorizadas por tipo de resposta. A etapa seguinte foi a análise vertical, observando-se as respostas de todos os respondentes para cada tipo de item analisado. Elas foram agrupadas, sendo possível dimensionar a concordância do grupo de respondentes com os tipos de resposta. As técnicas aplicadas foram análise de conteúdo (Krippendorff, 2004; Bardin, 2016).

A perspectiva longitudinal também foi explorada e analisada na mesma matriz. O corte utilizado foi o período de gestão de cada executivo responsável pela atividade. De 2012, criação da estrutura, até 2017, ela teve três gestores. Assim, foram considerados três momentos: marco 1 - 2012 e 2014; marco 2 - 2014 e 2016 e marco 3: a partir de 2016. Tal corte tem a ver com as diferenças marcantes em relação à atuação da estrutura dedicada e criação/utilização dos mecanismos. O roteiro de entrevistas foi composto por perguntas que remetiam a estes três momentos e os entrevistados eram os gestores e parceiros que estiveram presentes ao longo destes momentos. De acordo com o que prevê a pesquisa longitudinal focou-se no entendimento das mudanças nestes períodos, buscando-se identificar suas especificidades.

\subsection{O portfólio de alianças}

A empresa nódulo está há mais de 40 anos no mercado e adota uma estratégia de diferenciação, sendo reconhecida como de excelência em seu campo de atuação. Ao longo do tempo, investiu de maneira consistente no crescimento por meio de alianças - locais ou internacionais. Particularmente em relação ao conjunto de alianças analisadas, o foco do crescimento era regional, ou seja, pretendia-se alcançar as demais regiões do país afora RJ, SP e MG, onde já estava presente e dominava o mercado.

O projeto de expansão por meio de alianças teve início no ano 2000 e sua gestão se dava por meio de controles manuais e embrionários. Não havia formalização dos acordos por meio de contrato ou de atribuições de deveres e direitos. Não havia sistematização ou criação de regras para a atuação conjunta e as decisões eram analisadas caso a caso. Esse modus operandi perdurou até 2012, quando o número de parceiros cresceu drasticamente, aumentando a complexidade da operação e a necessidade de melhoria da sua estrutura de gestão.

A primeira ação foi criar uma área de gestão dedicada à relação com os parceiros. Cada um possuía particularidades regionais e, consequentemente, apresentava demandas 
específicas. A estrutura era composta por um gerente coordenador e uma analista de negócios e passou a ser a interface oficial que mediava a relação de todas as áreas de negócio com os parceiros. Essa estrutura estava ligada a uma diretoria.

O Escritório de Gestão dos Parceiros (EGP) formalizou as alianças por meio de contratos, que visavam a criação das bases para a atuação conjunta, os direitos e deveres, divisão de resultados e critérios para a relação estabelecida. Criava, também, segurança jurídica que permitia investimentos no objeto-fim das alianças - a ampliação do mercado de atuação.

Em 2012, dada as diferenças entre os parceiros e os diferentes níveis e conhecimento sobre as práticas e estratégia da empresa nódulo, o EGP passou a promover anualmente encontros de troca de conhecimento e melhores práticas e treinamento. Nesse ano foram formalizadas metas, estabelecidas conjuntamente entre os parceiros e a empresa nódulo.

Em 2014, a expansão regional estava consolidada e não foram estabelecidas novas alianças. Os anos seguintes foram de ajustes e aprofundamento das relações constituídas.

Em 2015, foi conduzida uma pesquisa $360^{\circ}$, na qual ambas as partes avaliaram o grau de satisfação com a estrutura e mecanismos estabelecidos. Na sequência, realizou-se um encontro, considerado pelos participantes um marco na relação e melhoria das interfaces entre os parceiros e a empresa nódulo. Denominado "Projeto Boa Distância", seu objetivo era estreitar e melhorar o relacionamento entre as partes e teve início com um encontro para discussão da parceria. $\mathrm{O}$ projeto se estendeu por mais de um ano e contou com representantes dos parceiros e da empresa nódulo.

Em 2017, foi estabelecida uma nova etapa de estruturação do relacionamento, com a adoção do sistema Freshdesk - uma plataforma de atendimento cujo objetivo principal era a gestão de solicitações e atendimento dos parceiros, possibilitando a definição de prioridades e acompanhamento das solicitações. A plataforma permitia a formalização das interações entre as partes, além da avaliação de metas e definição de um ponto único de contato na interação com a empresa nódulo.

A estrutura de gestão implementada em 2012 adotou, ao longo do tempo, quatro mecanismos de formação das capacidades de aliança: 1) sessões conjuntas de planejamento; 2) registro de melhores práticas, 3) intranet e 4) programas de treinamento in house.

\section{ANÁLISE}

Investigou-se quais são as vantagens e desvantagens percebidas pelos gestores da implementação de um escritório dedicado à gestão das alianças e quais os mecanismos de gestão criados por essa estrutura e com qual finalidade.

\subsection{Vantagens e desvantagens do Escritório de Gestão dos Associados}

A literatura aponta para a importância das estruturas dedicadas à gestão das alianças (Heimeriks \& Duysters, 2007; Kale et al., 2002) e como elas ajudam na construção das capacidades de aliança (Kale et al., 2002). A análise das entrevistas alinha-se a essas afirmações. Segundo um entrevistado da empresa nódulo sobre a criação da estrutura: "Foi com o objetivo de organizar essa expansão, criar critérios, definir regras e padrões. Então o papel era trazer regras, procedimentos, processos, desdobramento da estratégia da empresa para os parceiros, controle, atendimento e relacionamento".

Em relação às vantagens apontou-se para a possibilidade de organização e mediação da relação entre as partes - citado pela grande maioria dos parceiros:

Acho que a principal vantagem é você conseguir tratar todos de maneira igual. É 
padronizar essa relação [...], estabelecendo um canal que vai, ao longo do tempo, transmitindo para todos as mesmas coisas. Uniformizando comportamento, as atuações, as formas de trabalho.

Milagres et al. (2014) afirmam que uma estrutura dedicada estabelece os padrões de comportamento aceitos pelos gestores das alianças, , diminuindo os conflitos. Esse fato é corroborado no caso estudado: "Acho que essa ideia de ter alguém como canal era para tentar apaziguar e fazer uma espécie de elo entre o externo e o interno para tentar viabilizar projetos".

A análise dos documentos confirma a necessidade de padronização e organização. Na primeira fase foram identificados esforços do EGP para desenhar processos, formalizar a relação contratual, estruturar a aferição de resultados e estabelecimento de metas, montar treinamentos e encontros periódicos para o compartilhamento de conhecimentos e práticas.

Outra vantagem identificada refere-se à centralização das demandas, comprovando a literatura específica, que afirma que ela facilita a captura, integração e disseminação do conhecimento gerado nos processos de alianças (Kale et al., 2002). Conforme depoimento de um parceiro:

Outro aspecto que acho que motivou a criação desse ponto focal foi uma certa canalização das demandas. Na época, a empresa entendeu, eu achei acertado também, que seria importante, além de melhorarmos a interlocução, termos dentro desse canal de gestão dos parceiros uma forma de identificar demandas comuns, tentar desenvolver alguma política e melhorar processos. Então, uma das necessidades, além da melhoria da comunicação, foi tentar entender as demandas que estavam surgindo e como poderiam ser feitas ações para atendê-las demandas.

Em relação às desvantagens, embora com baixas ocorrências, os parceiros revelaram insatisfação com a lentidão na resolução de questões e certa burocratização. Particularmente, em relação à lentidão, afirmam que a autonomia do EGP é o aspecto central.

A desvantagem que eu vejo é que para algumas soluções rápidas, você diminuiu um pouco o tempo de resposta, porque acaba colocando um intermediário que não tem autonomia, ou a tem limitada.

Dois aspectos merecem ser mencionados em relação à posição do EGP. O primeiro é que ele ficou em posição intermediária entre os parceiros e as unidades de negócio, atuando muito mais como gestor das alianças do que como um depositário de conhecimento e sistematização de experiências apreendidas - diferentemente do que é apontado pela literatura (Heimeriks \& Duysters, 2007; Kale et al., 2002). O segundo aspecto, refere-se à autonomia. Heimericks et al. (2009) discutem a importância de a área ter respaldo, seja por meio de cargos, seja por conexão com o alto escalão da organização.

De maneira geral pode-se dizer que as seguintes vantagens/desvantagens foram enumeradas:

Tabela 1 - Vantagens x Desvantagens do Escritório de Alianças

\begin{tabular}{ll}
\hline \multicolumn{1}{c}{ Vantagens } & \multicolumn{1}{c}{ Desvantagens } \\
\hline $\begin{array}{l}\text { Organizar e mediar a relação entre os } \\
\text { parceiros }\end{array}$ & Lentidão na resolução de problemas \\
\hline Centralizar as demandas & $\begin{array}{l}\text { Incapacidade da área de resolver os } \\
\text { problemas da relação }\end{array}$ \\
\hline $\begin{array}{l}\text { Entender as diferentes características de } \\
\text { cada parceiro - customizar as soluções }\end{array}$ & $\begin{array}{l}\text { Não ter mais acesso direto a outras } \\
\text { unidades de negócio }\end{array}$ \\
\hline Fazer a gestão do conhecimento & Ser visto apenas como apoio \\
\hline
\end{tabular}




\begin{tabular}{ll}
\hline Ouvidoria para resolução de conflitos & Dificuldade de repassar a demanda \\
\hline
\end{tabular}

Fonte: os autores.

Contrariamente à literatura que aponta para a contribuição do escritório no que se refere à gestão do conhecimento, sistematização das experiências e disseminação do know how (Kale et al., 2002; Heimeriks \& Duysters, 2007; Heimeriks et al., 2009), apenas um parceiro mencionou essa vantagem. Este aspecto aponta para a dedicação do escritório voltada para a operação da relação e sua baixa maturidade/conhecimento sobre as funções clássicas apontadas na literatura.

\subsection{Instrumentos de gestão}

Segundo Heimeriks et al. (2009), existem quatro grandes categorias de soluções para a formação das capacidades de aliança: estrutura funcional, ferramentas de gestão de alianças, treinamento e contratação de terceiros. Afora a estrutura funcional, os demais são divididos em onze subcategorias, das quais apenas quatro instrumentos são citados/utilizados em algum grau: 1) sessões conjuntas de planejamento; 2) registro de melhores práticas, 3) intranet para gestão de recursos das alianças e 4) programas de treinamento in house.

\subsubsection{Sessões conjuntas de planejamento}

Heimericks et al. (2009) defendem que o compartilhamento de conhecimento e expectativas em sessões conjuntas de planejamento fazem com que os parceiros foquem nas estratégias das alianças e permitem maior troca de experiências quanto aos objetivos. No caso, os documentos mostram que as sessões conjuntas de planejamento aconteceram regularmente a partir de 2012, assim como encontros anuais.

Entretanto, as entrevistas não revelam os resultados elencados por Heimericks et al. (2009), já que os entrevistados afirmam que os encontros se restringiam à discussão sobre as metas e resultados. Criticaram a falta de planejamento conjunto, falta de acompanhamento do que era contratado e falta de gestão dos recursos para a entrega das metas. Na perspectiva dos gestores da empresa nódulo, o objetivo era construir as bases para formação e alinhamento de expectativas sobre os resultados esperados. Neste sentido, pode-se afirmar que o instrumento foi implementado parcialmente.

\subsubsection{Registro de melhores práticas}

Heimericks et al. (2009) definem que o registro e o estímulo à troca de experiências adquiridas estimulam o desenvolvimento das capacidades de aliança. No caso, apesar de não haver um processo sistematizado de troca de conhecimento, foram encontrados documentos e depoimento que mostram iniciativas neste sentido. "A troca de conhecimento é uma coisa. Eu acho essa troca incrível. Se você perguntar o que foi um ponto alto, é essa troca de conhecimento". Nas entrevistas pôde-se perceber a complementariedade de recursos e conhecimentos entre os parceiros e como esse registro facilitou a troca. Apesar disso, a maior parte das desvantagens apontadas se concentra neste quesito - pouca troca entre as partes.

Embora a literatura identifique que processos de codificação das melhores práticas são mecanismos importantes na formação de capacidades de aliança (Draulans et al., 2003), a análise dos documentos e entrevistas indicou baixa utilização desses instrumentos.

\subsubsection{Intranet para gestão de recursos das alianças}


A codificação das melhores práticas contribui para a difusão de informações sobre o progresso das alianças e, desse modo, permite aos gestores uma base de consulta e fonte de aprendizado (Heimericks et al., 2009). Na percepção dos respondentes, a ferramenta implementada teve por objetivo principal o controle. Apesar dessa diferença frente à literatura, os entrevistados afirmam que a ferramenta se constituiu em uma boa forma de gerenciar as demandas entre as partes. Ela padronizou o comportamento dos parceiros e reduziu o índice de erros, abrindo perspectivas para o aprendizado. Ressaltam, porém, que ela não substitui a relação próxima e pessoal, além de, por vezes, burocratizar o processo. Há consenso de que o instrumento tem espaço para melhorias, pois ainda é muito operacional e seu uso não oferece insumos para o desenvolvimento de conhecimento sobre as alianças.

\subsubsection{Programas de treinamento in house}

Heimericks et al. (2009) atestam que os treinamentos, de qualquer natureza, ajudam a esclarecer aspectos específicos da gestão das alianças e permitem o aprendizado a partir de experiências vividas pela organização. No caso específico, os treinamentos eram feitos em conjunto com os parceiros e foram utilizados para o desenvolvimento de capacidades para lidar com os conflitos inerentes às parcerias. Diferentemente do proposto na literatura, não foram feitas ações para que a organização nódulo se preparasse para gerir melhor suas alianças, ou seja, não foram feitos treinamentos específicos para os gestores envolvidos com as alianças. Os objetivos do treinamento eram voltados para melhorar a atuação dos parceiros e disseminação da cultura, processos, procedimentos e produtos da empresa nódulo. Além disto, foram utilizados para a construção de uma cultura de colaboração entre os parceiros.

$\mathrm{Na}$ perspectiva dos parceiros: "Eu considero os treinamentos importantíssimos e, sempre que tiver, vou mandar minha equipe, ou para se capacitar, ou para se atualizar. Acho fundamental a equipe sentir o ambiente da empresa nódulo, viver a cultura, vivenciar".

De maneira geral, pode-se afirmar que, apesar de terem sido encontrados neste caso quatro dos mecanismos descritos na literatura, os instrumentos utilizados pela empresa nódulo foram implementados parcialmente e, em sua maioria, de maneira diferente daquela proposta pela literatura. A empresa nódulo não aproveitou os instrumentos estabelecidos para o seu aprendizado. Ou seja, a implementação se deu no nível das alianças e foi muito utilizada na perspectiva do desenvolvimento do seu relacionamento com os parceiros.

Nas entrevistas não se percebeu preocupação em acumular e desenvolver as capacidades de aliança. Apesar de ter sido criado um escritório de gestão das alianças e aplicado muitos dos instrumentos sugeridos pela literatura, a empresa não foi capaz de absorver e internalizar o conhecimento. Usualmente, os estudos sobre capacidades de aliança são focados na importância das funções de aliança e nas ferramentas e mecanismos que as empresas podem adotar para o seu gerenciamento (Dyer et al., 2001; Heimeriks \& Duysters, 2007; Sluyts et al. 2010).

Entretanto, outra linha de autores, como Ziggers and Tjemkes (2010) defendem que as capacidades de aliança por si só não garantem resultados superiores, sendo necessário que elas sejam adequadamente colocadas em prática. Milagres et al. (2014) corroboram esse ponto quando destacam que as atitudes pró-cooperação são de vital importância, uma vez que os mecanismos de gestão por si só não garantem esse resultado.

\subsection{Perspectiva longitudinal - a estrutura sob as diferentes gestões}

O caso foi analisado em sua perspectiva longitudinal, considerando três marcos "Marco 1": criação do escritório até 2014; "Marco 2"- de 2014 a 2016 e "Marco 3" - a partir de 2016. O objetivo foi avaliar como a estrutura de gestão e os mecanismos implementados se 
desenvolveram ao longo do tempo na construção das capacidades de aliança. Isto porque, se o objetivo com uma estrutura e instrumentos específicos é melhorar a gestão das alianças, é preciso avaliar como se deu o aprendizado e sua utilização ao longo do tempo. Ademais, como a pergunta a ser respondida tem a ver com as vantagens de uma estrutura específica que se altera ao longo do tempo, fruto do aprendizado colhido, é preciso avaliar a percepção acerca dessas vantagens longitudinalmente.

Marco 1 - Segundo os respondentes, o objetivo principal do escritório de gestão era a mediação entre os parceiros e a empresa nódulo. No primeiro momento foi estruturada a relação. Tratava-se de um período de crescimento do número de alianças e, portanto, da complexidade da gestão do portfólio. A principal característica desse momento, sob a perspectiva da empresa nódulo, era estabelecer regras, procedimentos, manter o relacionamento baseado na confiança existente até então, mas melhorar o acompanhamento e o fortalecimento das relações. Para tanto, foi escolhido um gestor sênior. De maneira geral percebeu-se que a vantagem dessa escolha estava na organização e mediação do relacionamento. De acordo com um parceiro:

O gestor entra já com o intuito de começar essa cooperação, essa gestão. Ele passa a criar determinados padrões, a ficar muito próximo dos parceiros, passa a entender de forma bastante árdua a questão da regionalidade em um país continental. A fase desse gestor foi onde começou a estruturar isso.

A análise de documentos confirma a característica estruturante do "Marco 1", com a criação dos encontros anuais, treinamento básico dos parceiros e troca de conhecimento. Ainda que as iniciativas sejam incipientes na comparação com o que a literatura aponta como melhores práticas, havia uma percepção de movimento para fortalecer a cooperação entre as partes. As desvantagens percebidas se concentravam na falta de autonomia da estrutura para decidir.

Marco 2 - Neste momento, dada a importância dos parceiros para o crescimento da empresa nódulo, substitui-se o gerente sênior por um diretor, mudança percebida como um sinal da relevância dessas alianças. Em consequência, o processo de tomada de decisão foi acelerado. Este movimento por parte da empresa nódulo revela seu aprendizado, ou seja, o acúmulo de experiência do escritório de gestão levou à percepção de que, para se obter melhores resultados com as alianças, era preciso mais agilidade nas decisões e autonomia. Ademais, a experiência anterior confirmou o fato atestado pela literatura de que as relações colaborativas são conflituosas, marcadas por diferentes visões de mundo e objetivos. A desvantagem percebida foi a falta de tempo do diretor para cuidar dos detalhes necessários, ou seja, faltou estrutura na equipe interna da empresa nódulo.

Marco 3 - caracterizado pela mudança da presidência e dos diretores da empresa nódulo. Nesse movimento, a gestão do Escritório de Gestão foi designada para outro gestor, com dedicação exclusiva à função. Novamente, percebe-se que o escritório cumpriu seu objetivo, ou seja, a experiência anterior auxiliou na estruturação do escritório, por meio da formação de uma equipe. Em relação ás vantagens e desvantagens, as entrevistas revelaram que se percebia um aumento da profissionalização, das ferramentas, dos instrumentos etc. Mas, também, aumentou a burocratização e voltou-se a perceber falta de autonomia e de relevância, dada a substituição do diretor por um gerente. Além disso, vários instrumentos criados ao longo dos marcos 1 e 2 deixaram de ser utilizados.

Outro aspecto destacado foi o endurecimento da relação. O gestor que assumiu o escritório não possuía treinamento em gestão de alianças e o aprendizado colhido por seus antecessores não foi repassado. As relações que antes se estabeleciam por alto nível de confiança e diálogo foram substituídas por um relacionamento pautado pela autoridade. $\mathrm{Ou}$ 
seja, o escritório não foi capaz de sistematizar e transferir internamente o conhecimento acumulado. Embora apontado pela literatura como elemento importante na conformação das capacidades de aliança, não foi feito um treinamento interno. A consequência foi a perda do espírito de colaboração.

A análise longitudinal mostra que, apesar de a estrutura permanecer inalterada desde a concepção, sua atuação variou nos três marcos de análise, com uma percepção distinta de vantagens e desvantagens ao longo do tempo e com a utilização de mecanismos de formação de capacidades de alianças de forma diferente. Além disso, a análise parece corroborar os achados dos autores Milagres et al. (2014) e Ziggers and Tjemkes (2010), quando afirmam que melhores resultados na gestão das parcerias somente serão alcançados através da efetiva implementação das capacidades de aliança.

\section{CONCLUSÃO}

A literatura sugere uma correlação positiva entre as capacidades de aliança e a performance das empresas (Anand \& Khanna, 2000; Kale et al., 2002) e os mecanismos de transferência de conhecimento representam importante fator na mediação entre experiência e formação de capacidades de aliança (Slutz et al., 2008).

Nesse contexto, destaca-se a importância de estruturas dedicadas aos acordos cooperativos, assim como outros mecanismos que fazem a mediação entre a experiência em alianças e sua aplicação prática (Kale et al., 2002; Zollo \& Winter, 2002; Draulans et al., 2003; Dyer et al., 2001; Heimeriks \& Duysters, 2007; Kale \& Singh, 2007; Heimeriks et al., 2009).

Este artigo respondeu às seguintes perguntas:

Quais são as vantagens e desvantagens percebidas da implementação de um escritório dedicado à gestão das alianças?

Quais os mecanismos de gestão criados por essa estrutura e com qual finalidade?

De acordo com os entrevistados, a implantação do Escritório de Gestão apresenta mais vantagens que desvantagens. As vantagens são a organização, mediação e centralização do relacionamento, o que corrobora os estudos apresentados na literatura que entendem as funções dedicadas com um papel de coordenação das atividades relacionadas a alianças e que podem aumentar a habilidade das empresas para gerar maiores retornos nas alianças (Kale et al., 2002).

Entretanto, foram apresentadas, também, desvantagens, como a lentidão na tomada de decisões e burocratização. Isto porque a estrutura criada, na maior parte de sua existência, não tinha autonomia para resolução dos problemas, funcionando como um intermediário entre os parceiros e as unidades de negócio, sem agregar valor à relação. Nesse contexto, a estrutura executa um papel de repasse de informações, com perda de velocidade na resolução dos problemas. Este é um aspecto importante, uma vez que a literatura até então se restringiu a apontar para as vantagens da criação de uma estrutura dedicada à gestão do portfólio de parcerias.

Dois pontos são relevantes para a prática gerencial: a importância do estabelecimento de estruturas dedicadas e a necessidade de que elas tenham posição relevante na estrutura organizacional, obtendo desta maneira apoio interno e autonomia. O segundo aspecto é que, no caso analisado, o escritório interferiu na dinâmica do negócio, ou seja, a função do EGP deveria ter se restringido a se constituir como um lócus para o acúmulo, criação de experiências e capacidades de aliança, conforme sugerido pela literatura, e não em um gestor dos negócios das alianças.

Em relação aos instrumentos, o EGP implementou quatro, dentre os descritos na literatura. A análise das respostas parece indicar que, apesar de a utilização desses 
mecanismos sugerir uma estratégia de cooperação, as iniciativas foram incipientes e não refletiram na sua totalidade os conceitos descritos pelos autores. Um exemplo pode ser observado nas sessões conjuntas de planejamento. Zollo and Winter (2002) afirmam que o processo de planejamento conjunto pode oferecer ocasiões propícias para os gestores identificarem conexões entre ações e resultados e focarem naquelas que mais contribuem para os resultados conjuntos. $\mathrm{Na}$ análise das entrevistas, o planejamento conjunto se limitou à definição de metas para avaliar a performance dos parceiros, mas não se estendeu para a identificação de ações e conexões entre ações e resultados. Consequentemente, não se constituiu em instância para acúmulo, criação e compartilhamento de conhecimento. A mesma conclusão se aplica aos demais itens implementados, levando à proposição de que a criação do EGP por si só não garante a implementação dos mecanismos de desenvolvimento de capacidades de aliança, assim como não garante que eles sejam implementados na sua totalidade.

A análise longitudinal suporta essa conclusão. A criação do escritório é vista no "Marco 1" como um facilitador da relação, implementando processos e fluxos de trabalho e se tornando o ponto focal da relação. Entretanto, a estrutura também é percebida com baixa autonomia para decisão, e a análise dos documentos mostra que apenas três dos onze mecanismos de formação das capacidades mencionados por Heimeriks et al. (2009) foram implementados. No "Marco 2", com a transição da liderança, há uma percepção de upgrade no nível de interlocução, com aumento da importância dos parceiros e decisões mais rápidas. Entretanto, percebeu-se a falta de tempo dedicado. Os mecanismos implementados no momento anterior foram mantidos.

No "Marco 3" há uma percepção de downgrade do nível de interlocução, com perda de prestígio dos parceiros e maior demora na resolução dos problemas. Há, também, a percepção de profissionalização da gestão, mas todas as iniciativas de relacionamento são ofuscadas pelo processo de renegociação das remunerações.

Logo, a análise longitudinal parece mostrar que no "Marco 1" houve a tentativa de desenvolver processos internos de aprendizado e experiência, conforme descrito por Draulans et al. (2003), além da disseminação desses conhecimentos dentro da organização, mesmo que de forma incipiente. Tal estratégia aparentemente foi mantida no "Marco 2", mas abandonada no "Marco 3" em função da incapacidade do escritório de treinar os novos gestores e repassar toda a experiência e conhecimentos acumulados. A relação se deteriorou, passando de uma lógica de cooperação, com mais trocas de conhecimento entre as partes, para um modelo mais comercial, voltado para as atividades operacionais da aliança. Esse aspecto corrobora o que o estudo de campo de Milagres et al. (2014) identificou, no qual a estrutura por si só não garante a manutenção das estratégias de cooperação.

Em resumo, a pesquisa de campo corrobora a literatura sobre a importância das estruturas de gestão dedicadas, nas quais a mediação, a organização e a centralização da relação são os principais pontos positivos. Entretanto, este trabalho avança ao apontar desvantagens, como burocratização, lentidão, afastamento das áreas fins de negócio, ou seja, o escritório atuando como um intermediário que retarda a tomada de decisão.

É importante caracterizar, entretanto, as especificidades do escritório de parcerias analisado. A opção da empresa nódulo foi a de trabalhar o desenvolvimento das capacidades de aliança em conjunto com os parceiros. Assim, coube ao escritório não só acumular e disseminar o conhecimento adquirido, mas, também, e, principalmente, gerenciar a relação. Diferentemente do caso estudado por Milagres et al. (2014) em que o escritório possuía as funções clássicas apontadas pela literatura, atuando como depositário e disseminador interno do aprendizado colhido. 


\subsection{Contribuições, limitações e sugestões de pesquisa futura}

Esta pesquisa apresenta contribuições para a discussão sobre o papel do escritório de gestão de alianças e as relações entre ele e os demais mecanismos de formação das capacidades de aliança. Contribui ao trazer base empírica para o entendimento de que o escritório dedicado é fator importante no desenvolvimento das capacidades de aliança. Milagres (2012) cita a contribuição de diversos autores que avaliam a geração de conhecimento e novas oportunidades de parceria como resultados importantes do processo de gestão do portfólio de alianças. Entretanto, pouco foi estudado sobre as desvantagens percebidas da atuação dessa estrutura.

Além disso, contribui ao mostrar as inter-relações entre o escritório e os demais mecanismos de formação das capacidades de alianças citadas por Heimeriks et al. (2009), detalhando a finalidade desses instrumentos e o grau de sua implementação.

Outra contribuição está relacionada à avaliação longitudinal da atuação de um escritório de alianças elucidando a evolução da estrutura, detalhando de que forma o conhecimento foi ou não internalizado e como ela é contexto dependente. Ressalta, ainda, questões a serem observadas pelas empresas que optam por ter uma estrutura específica, como a necessidade de autonomia e apoio organizacional à estrutura dedicada, além da importância de treinamento específico dos atores envolvidos em gestão de portfólio de alianças. Ainda em relação à prática, o estudo traz evidências sobre a operação do escritório e instrumentos utilizados, auxiliando deste modo, os gestores envolvidos em parcerias.

Em relação às limitações, encontra-se o uso de estudo de caso único. Yin (2015) afirma que, apesar de o estudo de caso permitir maior compreensão sobre o tema, com foco nos "porquês" e "comos", um estudo desse tipo limita a generalização dos achados. Outra limitação se refere ao processo de levantamento dos dados, calcado nas percepções e memória dos entrevistados. Tais percepções podem ter vieses. Tendo em vista esse cenário de contribuições e limitações, sugere-se como tema de novas pesquisas o aprofundamento das causas de falha na implementação dos mecanismos de formação das capacidades de aliança por parte das estruturas dedicadas à gestão das alianças.

Este trabalho avançou também apontando que a estrutura por si só não garante a estratégia de cooperação, mas não mostra as principais barreiras para esse resultado. Podem ser levantadas hipóteses, como mudança da estratégia da alta liderança, desconhecimento dos gestores sobre os atributos de um processo cooperativo, o perfil da liderança ou questões puramente econômicas, mas não há elementos suficientes que embasem essas considerações.

Outro caminho para futuras pesquisas é mapear quantitativamente a influência da adoção dos mecanismos de formação das capacidades de aliança entre si. Apesar de as vantagens identificadas neste trabalho corroborarem a literatura e iluminarem as inter-relações entre eles, não há uma hierarquização entre os diferentes mecanismos. 


\section{REFERÊNCIAS}

ANAND, Bharat N; KHANNA, Tarun. Do firms learn to create value? The case of alliances. Strategic Management Journal, Boston, v. 21, p. 295-315, 2000.

BARDIN, Laurence. Análise de conteúdo. São Paulo: Edições 70, 2016.

CASTRO, Ignacio; ROLDÁN, José L. Aliiance portfolio management: dimensions and performance. European Management Review, New York, v. 12, p. 63-81, 2015.

COOLS, Kees; ROOS, Alexander. The role of alliances in corporate strategy. Organization Science, Boston, v. 13, n. 3, p. 339-351, 2005.

DRAULANS, Johan; DEMAN, Ard-Pieter; VOLBERDA, Henk W. Building alliance capability: management techniques for superior alliance performance. Long Range Planning, Amsterdã, v. 36, p. 151-166, 2003.

DUYSTERS, Geert et al. Do firms learn to manage alliance portfolio diversity? The diversity-performance relationship and moderating effects of experience and capability. European Management Review, Eindhoven v. 9, p. 139-152, 2012.

DYER, Jeffrey H.; KALE, Prashant; SINGH, Harbir. How to make strategic alliances work. Sloan Management Review, Boston, v. 42, p. 37-43, 2001.

DYER, Jeffrey H.; SINGH, Harbir. The relational view: cooperative strategy and sources of interorganizational competitive advantage. Academy of Management Review, Philadelphia, v. 23, p. 660-679, 1998.

EISENHARDT, Kathleen M. Building theories from case study research. Academy of Management Review, Boston, v. 14, n. 4, p. 532-550, 1989.

FIGGIS, Patrick; BARNES, Kelly; ARNOLD, Dean. A new treatment?: healthcare findings from the 20th Annual Global CEO Servey. London: PwC, 2017.

FLICK, Uwe. Uma introdução à pesquisa qualitativa. Porto Alegre: Bookman, 2004.

GULATI, Ranjay. Network location and learning: the influence of network resources and firm capabilities on alliance formation. Strategic Management Journal, Chicago, v. 20, p. 397-420, 1999.

GULATI, Ranjay. Does familiarity breed trust? The implications of repeated ties for contractual choice in alliances. Academy of Management Journal, Chicago, v. 38, p. 85-112, 1995.

HEIMERIKS, Koen H.; BINGHAM, Christopher B.; LAAMANEN, Tomi. Unveiling the temporally contingent role of codification in alliance success. Strategic Management Journal, Copenhagen, n. 36, p. 462-473, 2015.

HEIMERIKS, Koen H.; DUYSTERS, Geert. Alliance capability as a mediator between experience and alliance performance: an empirical investigation into the alliance capability development process. Journal of Management Studies, Copenhagen, v. 44, p. 25-49, 2007. 
HEIMERIKS, Koen H.; KLIJN, Elko; REUER, Jeffrey J. Building capabilities for alliance portfolios. Long Range Planning, Copenhagen, v. 42, p. 96-114, 2009.

HEIMERIKS, Koen H.; SCHREINER, Melanie. Relational quality, alliance capability, and alliance performance: an integrated framework. In: SANCHEZ, Ron; HEENE, Aime (ed.). Enhancing competences for competitive advantage - advances in applied business strategy. Bingley: Emerald Group, 2010. v. 12, p. 145-171.

IRELAND, R. Duane; HITT, Michael A.; VAIDYANIATH, Deepa. Alliance management as a source of competitive advantage. Journal of Management, New York, v. 28, p. 413-446, 2002.

KALE, Prashant; SINGH, Harbir. Managing strategic alliances: what do we know now, and where do we go from here? Academy of Management Perspectives, Philadelphia, v. 23, p. 45$62,2009$.

KALE, Prashant; SINGH, Harbir. Building firm capabilities through learning: the role of the alliance learning process in alliance capability and firm-level alliance success. Strategic Management Journal, Philadelphia v. 28, p. 981-1000, 2007.

KALE, Prashant; DYER, Jeffrey H.; SINGH, Harbir. Alliance capability, stock market response, and long-term alliance success: the role of the alliance function. Strategic Management Journal, Philadelphia, v. 23, p. 747-767, 2002.

KOHTAMAKI, Makro; RABETINO, Rodrigo; MOLLLER, Kristian. Alliance capabilities: A systematic review and future research directions. Industrial Marketing Management, Oxford, v. 68, p. 188-201, 2018.

KRIPPENDORFF, Klaus. Content analysis: an introduction to its methodology. $2^{\text {nd }}$ ed. Thousand Oaks, CA: Sage Publications, 2004.

LITTLE, Arthur Dehon. Partnering: challenges for the old and new economy. 2001. Apresentação. Disponível em: www.adlittle.de/asp/orderpublications.asp?ID=28. Acesso em: 02 jun. 2018.

Arthur D. Little, 2001, Partnering. Challenges for the old and new economy. FactsAssessments-Recommendations based on a global Arthur D. Little survey (September 2001), http://www.adlittle.de/asp/orderpublications.asp?ID=28.

MILAGRES, Rosileia. O desenvolvimento de capacidades em gestão de alianças: Uma revisão da literatura. Revista Portuguesa e Brasileira de Gestão, Lisboa, v. 12, n. 1, 2012.

MILAGRES, Rosileia; REZENDE, Otávio. Estratégia de cooperação - alianças entre organizações e convergências de objetivos. In: MENDES, Luis Augusto Lobão; ONO, Mauri Seiji (org.). Conselho estratégico: guia da alta administração para o desenvolvimento da estratégia corporativa. São Paulo: Saraiva, 2014. p. 177-211.

MILAGRES, Rosileia; REZENDE, Otávio; GONÇALVES, Carlos Alberto. Capabilities as a necessary Sed Non Sufficiens condition for Portfolio Management of Strategic Alliances The Embrapa Case. Revista Gestão \& Tecnologia, São Paulo, v. 14, n. 2, p. 25-50, 2014. 
MOLLER, Kristian. Theory map of business marketing: relationships and networks perspectives. Industrial Marketing Management, Oxford, v. 4, n. 3, p. 324-335, 2013.

NIESTEN, Eva; JOLINK, Albert. The impact of alliance management capabilities on alliance attributes and performance: a literature review international. Journal of Management Reviews, London, v. 17, p. 69-100, 2015.

SAMPSOM, Rachelle C. R\&D alliances and firm performance: The impact of technological diversity and alliance organization on innovation. Academy of Management Journal, New York, v. 50, p. 364-386, 2007.

SARKAR, M. B.; AULAKH, Preet S.; MADHOK, Anoop. Process capabilities and value generation in alliance portfolios. Organization Science, London, v. 20, p. 583-600, 2009.

SCHILLING, Melissa. Technology shocks, technological collaboration, and innovation outcomes. Organization Science, New York, v. 26. p. 668-686, 2015.

SCHREINER, Melanie; KALE, Prashant; CORTSTEN, Daniel. What really is alliance management capability and how does it impact alliance outcomes and success? Strategic Management Journal, London, v. 30, p. 1395-1419, 2009.

SLUYTS, Kim et al. Building capabilities to manage strategic alliances. Industrial Marketing Management, Cambridge, v. 40, n. 6, p. 875-886, 2011.

SLUYTS, Kim; MARTENS, Rudy; MATTHYSSENS, Paul. How to build alliance capability: a life cycle approach. In: SANCHEZ, Ron; HEENE, Aime (ed.). Enhancing competences for competitive advantage - advances in applied business strategy. Bingley: Emerald Group, 2010. v. 12, p. 173-200.

SLUYTS, Kim; MARTENS Rudy; MATTHYSSENS, Paul. Towards a dynamic concept of alliance capability. University of Antwerp Department of Management, Antwerp, v. 13, 2008.

TZOKAS, Nikolaos; KIM, Young Ah; AKBAR, Hammad; AL-DAJANI, Haya. Absorptive capacity and performance: the role of customer relationship and technological capabilities in hightech. SMEs. Industrial Marketing Management, Oxford, v. 47, p. 134-142, 2015.

ZIGGERS, Gerrit W.; TJEMKES, Brian. Dynamics in Inter-firm collaboration: The impact of alliance capabilities on performance. International Journal on Food System Dynamics, Amsterdam, v. 1, n. 2, p. 151-166, 2010.

ZOLLO, Maurizio; WINTER, Sidney G. Deliberate learning and the evolution of dinamic. 2002. Organization Science, Paris, v. 13, n. 3, p. 339-351, May/June 2002.

Anexo:

Roteiro de Entrevistas 
O roteiro de entrevistas foi composto por perguntas para dois públicos: 1) Gestores do Escritório de Alianças e 2) Parceiros. Em ambos os casos o roteiro foi construído para auxiliar o entrevistado a se lembrar dos pontos avaliados como mais importantes sobre a história do projeto, abrangendo os três marcos do estudo longitudinal.

Questionário Semi Estruturado:

1. Na percepção do entrevistado, como foi a decisão pela criação de uma área dedicada à gestão dos Parceiros? Contexto da época, motivações, ganhos esperados e riscos envolvidos.

2. Qual era e estrutura dessa área? Quais eram seus principais objetivos e funções? Qual era o papel da empresa e do Parceiro - explorar os aspectos que caracterizam uma aliança (ex: objetivos compartilhados, alinhamento de atuação).

3. Quais são as vantagens e desvantagens percebidas pelos respondentes da implementação de uma estrutura dedicada à gestão dos Parceiros?

4. Que tipo de instrumentos de gestão eram utilizados para o alcance dos objetivos?

5. Como o entrevistado vê a adoção de um contrato formal com os Parceiros? Qual a finalidade? Vantagens e desvantagens?

6. Como o entrevistado vê a adoção de metas conjuntas? Qual a finalidade? Vantagens e desvantagens?

7. Como o entrevistado vê os encontros anuais com os Parceiros? Qual a finalidade desses encontros? Vantagens e desvantagens?

8. Como o entrevistado vê a adoção de treinamentos formais dos Parceiros e suas equipes? (Ex. programa básico). Quais os benefícios e riscos dessa ação?

9. Como o entrevistado percebe a regulamentação das normas para treinamento formal dos Parceiross e suas equipes? Quais os benefícios e riscos dessa ação?

10. Como o entrevistado percebe a realização de pesquisa avaliação do relacionamento entre as partes? Qual a finalidade dessa pesquisa? Vantagens e desvantagens?

11. Como o entrevistado percebe a introdução de uma ferramenta para registro das interações entre as partes? Qual a finalidade? Vantagens e desvantagens?

12. Na percepção do entrevistado, como é o processo de aprendizagem entre as partes?

13. Como o entrevistado vê os três momentos de gestão da estrutura? Há diferenças significativas de atuação entre elas? 